\title{
A method for the characterization of the reflectance of anisotropic functional surfaces
}

Regi, Francesco; Nielsen, J B; Li, Dongya; Zhang, Y; Frisvad, Jeppe Revall; Aanæs, Henrik; Tosello, Guido

Published in:

Surface Topography: Metrology and Properties

Link to article, DOI:

10.1088/2051-672X/aac373

Publication date:

2018

Document Version

Peer reviewed version

Link back to DTU Orbit

Citation (APA):

Regi, F., Nielsen, J. B., Li, D., Zhang, Y., Frisvad, J. R., Aanæs, H., \& Tosello, G. (2018). A method for the characterization of the reflectance of anisotropic functional surfaces. Surface Topography: Metrology and Properties, 6(3), [034005]. https://doi.org/10.1088/2051-672X/aac373

\section{General rights}

Copyright and moral rights for the publications made accessible in the public portal are retained by the authors and/or other copyright owners and it is a condition of accessing publications that users recognise and abide by the legal requirements associated with these rights.

- Users may download and print one copy of any publication from the public portal for the purpose of private study or research.

- You may not further distribute the material or use it for any profit-making activity or commercial gain

- You may freely distribute the URL identifying the publication in the public portal 


\title{
A method for the characterization of the reflectance of anisotropic functional surfaces
}

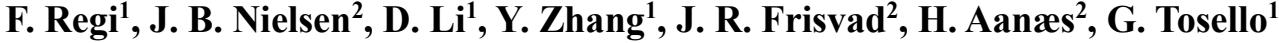 \\ ${ }^{1}$ Technical University of Denmark, Department of Mechanical Engineering, \\ ${ }^{2}$ Technical University of Denmark, Department of Applied Mathematics and Computer \\ Sciences, \\ E-mail: fraregi@mek.dtu.dk
}

\begin{abstract}
The functional properties of micro-structured surfaces have gained increasing interest thanks to many applications such as wetting, adhesion, thermal and/or electrical conductivity. In this study, directional optical properties, i.e. contrast between two regions of a surface, were achieved with an anisotropic microstructure composed of a close array of ridges. The anisotropic surface, designed as a combination of ridges, has been milled on a steel bar and replicated through hot embossing of Acrylonitrile butadiene styrene (ABS) and through replica technology using silicone rubber. The directional reflectance of the surface for a range of design-specific view-illumination configurations was determined using a method that involves a Hirox RH-2000 digital microscope, used as a gonioreflectometer. This method allows the empirical determination of the optimum surface microstructure for maximizing contrast between two horizontally orthogonal views. The results show that even if the uncertainty related to the instrumentation is up to $20 \%$ in some cases, this procedure is suitable for the characterization of the surface of both metal and plastic counterpart.
\end{abstract}

Keywords: micro-structure, functional surfaces, anisotropic reflectance, contrast

\section{Introduction}

The functional properties of micro-structured surfaces have gained increasing interest thanks to many applications in various products used in markets ranging from consumer goods to life sciences [1]. These properties are often limited by and related to the production method of the structure: functional master structures can be produced by means of micro-machining [2], diamond chiseling [3], laser ablation [4] or lithographic methods [5]. Then, the master structure can be replicated by means of techniques like hot and roll embossing or thermoforming [6], but the most important method for mass replication remains injection moulding [7].

The functionalities that may be achieved range from tribology properties [8], i.e. reducing friction between lubricated sliding surfaces [9], to microfluidic diagnostic devices [10], sensors [11], and optical structures. The last qualifies as one of the biggest markets. Many applications are about colour generation. Saito [12] provided examples of biomimetic inspired design approaches to obtain structural colour, which has a variety of potential applications because of its long-term resistance to discoloration and its pigment-free nature. In other fields, anti-reflective properties are required to increase the share of transmitted light, to improve the contrast of displays, and to avoid the formation of ghost images in imaging and illuminating systems [13, 14]. Similarly, the modelling of surface reflectance is a key area of study within several fields, including computer graphics where it is one of the main components that determines appearance. Weyrich in [15] proposes a system for manufacturing physical surfaces that, in aggregate, exhibits a desired surface appearance.

Furthermore, widespread approaches use arrays of asymmetric surface features, such as arrays of grooves, inclined pillars, and helical protrusions, to impart unique anisotropy in optical properties [16]. In this study, directional optical properties were achieved with a microstructure composed of a close array of ridges (figure 1), as described by Li in [17]. The scope was to create a surface with custom reflectance functions, similar to those obtained by Levin in [18] but using durable micro-milled structures. In particular, the purpose was to maximize the reflectance from a certain point of view and minimize it from its orthogonal position (figure 2). Using this principle, two adjacent orthogonally oriented arrays of ridges would generate a black and white contrast effect. This is referred to as orthogonal contrast.

This is an author-created, un-copyedited version of an article accepted for publication/published in Surface Topography: Metrology and Properties. IOP Publishing Ltd is not responsible for any errors or omissions in this version of the manuscript or any version derived from it. The Version of Record is available online at https://doi-org.proxy.findit.dtu.dk/10.1088/2051-672X/aac373 
However, in order to verify the functionality of the surface, there was a need for an accurate evaluation of the optical properties of the geometry, in particular the reflectance of the surface for a specific angle and tilting. For this evaluation, a method involving a Hirox RH-2000 digital microscope used as a gonioreflectometer has therefore been developed.
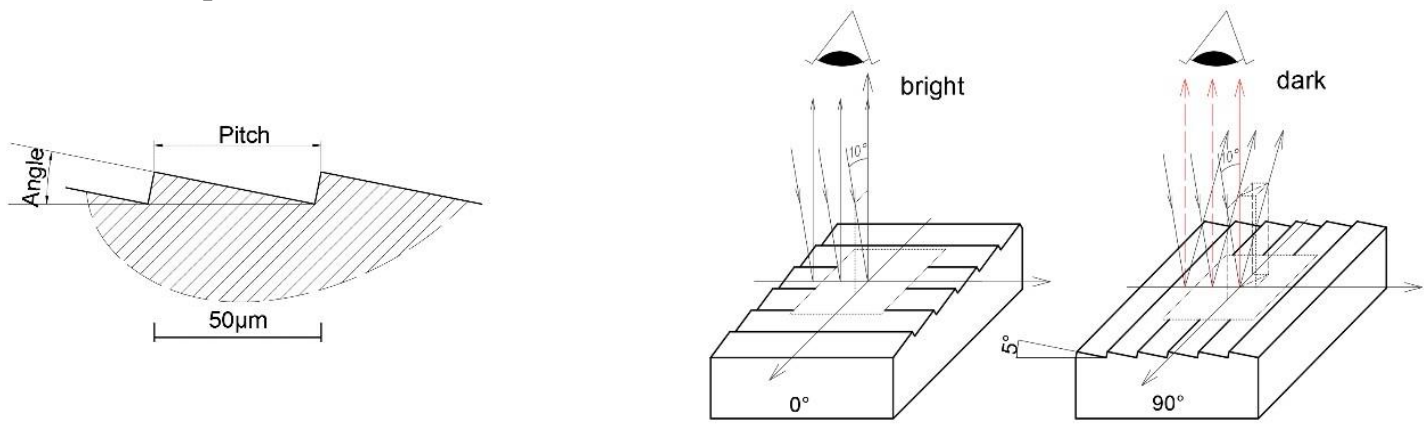

Figure 1. Anisotropic structure geometry: definition of pitch and angle.

Figure 2. Demonstration of orthogonal contrast generation.

\section{Experimental Methods}

The evaluated sample was composed of four different anisotropic surfaces, designed as a combination of ridges with length $50 \mu \mathrm{m}$ and defined by their angle in respect to the surface, 5, 10,15 and 20 degrees. Each surface has a dimension of $2 \mathrm{~mm}$ by $2 \mathrm{~mm}$.

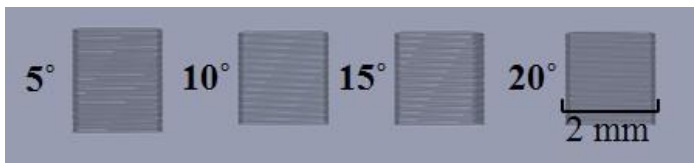

Figure 3. Schematic of the 4 anisotropic surfaces considered in this study.

The geometry has been milled on a steel bar by a precision five-axis milling machine. The milling parameters have been chosen experimentally and optimized accordingly [17]. Its replicas were obtained through replica technology using silicone rubber [19] and hot embossing of Acrylonitrile butadiene styrene (ABS). In the former, Accutrans AB Polyvinylsiloxane by COLTENE Holding AG was poured directly on the surface, pressed and cured for 5 minutes, before being carefully removed to avoid strain.

In the latter, the replication took place using Terluran RP-35 ABS by Ineos Styrolution on a Hydraulic Unit by Carver, Inc. The plates were kept at $220^{\circ} \mathrm{C}$ and pressed at $16 \mathrm{MPa}$ for 30 seconds.

\section{Measurement Method}

The radiometric measurements were carried out using a Hirox RH-2000 digital microscope operated as a gonioreflectometer, allowing for observing a sample under varying inclinations and azimuthal angles. This

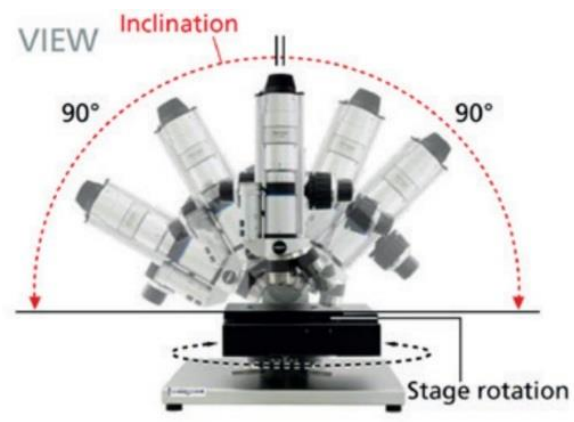

Figure 4. Schematic of the microscope's degrees of freedom. It allows an inclination $\theta$ and an azimuthal rotation $\varphi$. 
was achieved through the microscope's built in tilting functionality combined with a rotation stage, as shown in figure 4.

In addition, the microscope was modified to hold a constant LED based light-source at a fixed baseline relative to the optics, ensuring a constant camera-light-source angle; in the Rusinkiewicz parametrization [20], this angle is referred to as $\theta_{\mathrm{d}}$. This particular setup was created to simulate the scenario of a smartphone observing the surface under the illumination of the camera LED flash.

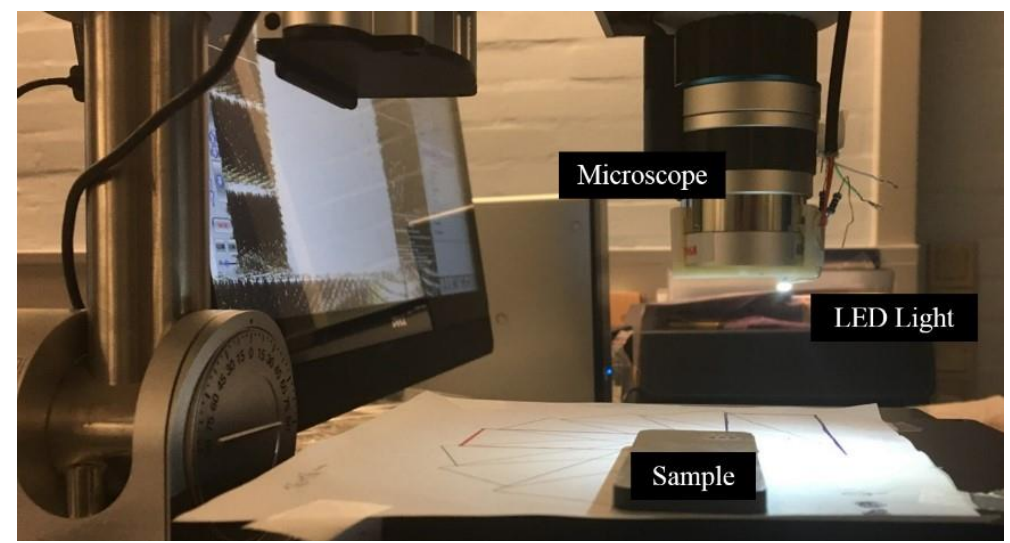

Figure 5. Microscope modified to hold a constant LED based light-source at a fixed baseline relative to the optics.

To obtain reflectance measurements from a CCD camera, the radiometric quantities are needed instead of the original pixel values obtained from the microscope camera. A conversion from pixel values to radiant exposure $\left(\mathrm{J} / \mathrm{m}^{2}\right.$ up to an unknown scaling factor $\left.k\right)$ is possible using high dynamic range (HDR) imaging. This means taking multiple images with different exposure times and merging them to a single image with radiant exposure values of floating point precision [21]. Debevec and Malik [21] carefully justify the use of multiple exposures to obtain radiometric image values. Their method involves measuring the camera CCD response function and using this as a weighting function when doing the merging. In practice, three images at predefined exposure times, or stops, are captured. Each image with a shorter exposure time covers the part of the radiant exposure range that is clamped by the CCD in the image with longer exposure time.

Since the same light source is used for all images, the unknown scaling factor $k$ will be the same and only the reflectance will change with the inclination and azimuthal angles. Reflectance values can thus be compared by inspecting differences in radiant exposure values between HDR images of different configurations. The contrast between two surfaces, or two orthogonal views of the same surface, can therefore be measured by calculating the difference between the respective HDR image values.

\section{Application}

It is important to define a few parameters:

- Each sample is defined by its ridge angle (figure 3);

- $\theta$ refers to the tilting angle of the microscope (inclination in figure 4-5);

- $\varphi$ is the azimuthal rotation of the sample about its central axis. (stage rotation in figure 4).

\subsection{Uncertainty Evaluation}

The milled metal sample has been used to evaluate the uncertainty related to the measurements.

In order to define the uncertainty, the microscope has been kept perpendicular to the surface $(\theta=\{0\})$, while the samples, the surfaces defined by angle 5 and angle 10 , have been rotated for half a circumference $(\varphi=$ $\{0 ; 45 ; 90 ; 135 ; 180\})$.

There is not a standard that defines the components of the uncertainty on this measurement method. Therefore, a type A uncertainty evaluation as prescribed by GUM [22] has been used:

$$
U=k \cdot u
$$

Where $\mathrm{U}$ is the expanded uncertainty, $\mathrm{k}$ is the coverage factor and 


$$
u=h \cdot \sqrt{\frac{\sum_{1}^{n}\left(\frac{\sum_{1}^{n} x_{i}}{n}-x_{i}\right)^{2}}{n \cdot(n-1)}} .
$$

The latter is the standard deviation of the mean value of a number $\mathrm{n}$ of measurement results $\mathrm{x}$, corrected by a safety factor $h$.

Six measurements have been taken for each of the ten selected positions, which are reported in table 1. The table also reports the average radiance values and the relative calculated percentage uncertainty, obtained applying a safety factor of 1.3 and considering a coverage factor of 2 (confidence level of $95 \%$ ).

Table 1. Summary of the average radiant exposure values at the selected positions

\begin{tabular}{ccc}
\hline Sample & Average $\left(\mathrm{kJ} / \mathrm{m}^{2}\right)$ & $\mathrm{U}(\%)$ \\
\hline ridge $5-\varphi 0$ & 74 & $8 \%$ \\
ridge $5-\varphi 45$ & 59 & $2 \%$ \\
ridge $5-\varphi 90$ & 66 & $3 \%$ \\
ridge $5-\varphi 135$ & 105 & $8 \%$ \\
ridge $5-\varphi 180$ & 182 & $21 \%$ \\
\hline ridge $10-\varphi 0$ & 45 & $1 \%$ \\
ridge $10-\varphi 45$ & 54 & $1 \%$ \\
ridge $10-\varphi 90$ & 63 & $2 \%$ \\
ridge $10-\varphi 135$ & 61 & $2 \%$ \\
ridge $10-\varphi 180$ & 70 & $5 \%$ \\
\hline
\end{tabular}

Probable causes of the uncertainty are the scattering of the light from the surface and the errors due to the manual handling of sample and instrumentation.

It is evident that the uncertainty rises with the increase of the radiance. The highest uncertainty, 21 percent of the average exposure, was given by the configuration "ridge angle $5-\varphi 180$ ", in which most of the light is reflected back to the microscope's lens. Nonetheless, the p-values from the paired t-tests between all the configurations were below 0.05 meaning significant differences between the means, hence the method was successful in the characterization of the trend.

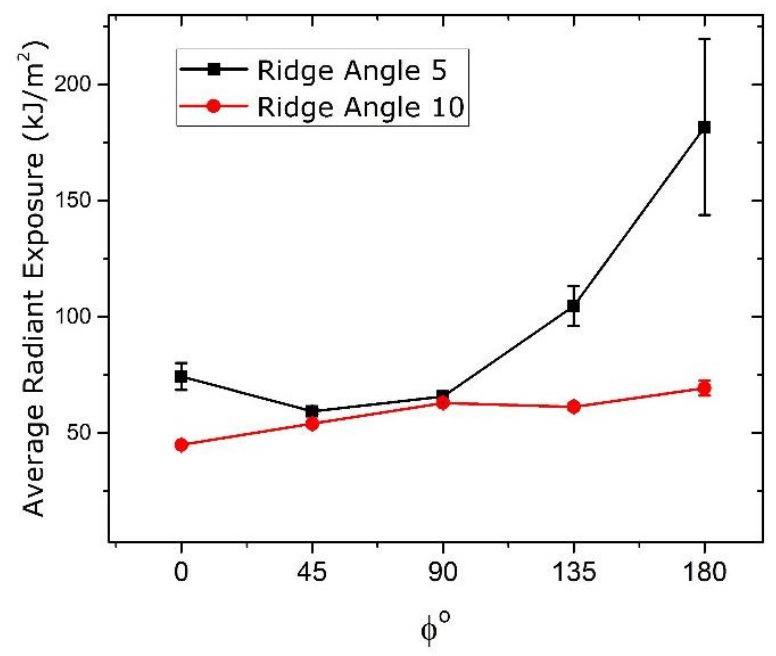

Figure 6. Graph depicting the radiant exposure and the uncertainty at the selected positions. 


\subsection{Functionality on different plastic materials}

Four samples in the two different materials and exhibiting diverse colours have been produced (table 2): two samples in ABS, dark green and blue, and two in silicone, light green and brown.

The inclination of the microscope $\theta$ ranged $\pm 20^{\circ}$, while the samples were placed at azimuthal angles $\varphi=\{0$, $90,180\}$

Table 2. Summary of the colours and materials of the considered samples.

\begin{tabular}{ccc}
\hline Sample & Material & Colour \\
\hline S5 & ABS & Dark Green \\
S6 & ABS & Blue \\
S9 & Silicone & Light Green \\
S11 & Silicone & Brown \\
\hline
\end{tabular}

The analysis of the collected data was focused on the determination of:

- Preferable ridge angle that maximazes the contrast between perpendicular structures;

- Colour and material that maximizes the contrast;

- Colour and material that gives the highest reflectance.

Figure 7 shows the data means analysis of the reflectance, while figure 8 presents a similar study on the contrast. The plots report the means for each value of a selected variable for every collected response. The vertical direction of the microscope $(\theta=0)$ produced the highest average reflectance, while the tilting strongly reduced it (figure 7). For what concerns the ridge, smaller ridge angles are preferred: the 10-degree ridge gave the best solution also in terms of contrast (figure 8). Finally, blue and light green had the highest absolute reflectance (figure 7), but performed poorly in terms of contrast (figure 8), and while the difference is small, ABS guarantees a better contrast. The directionality of the geometry makes the contrast $0^{\circ}-90^{\circ}$ stronger than the $90^{\circ}-180^{\circ}$.

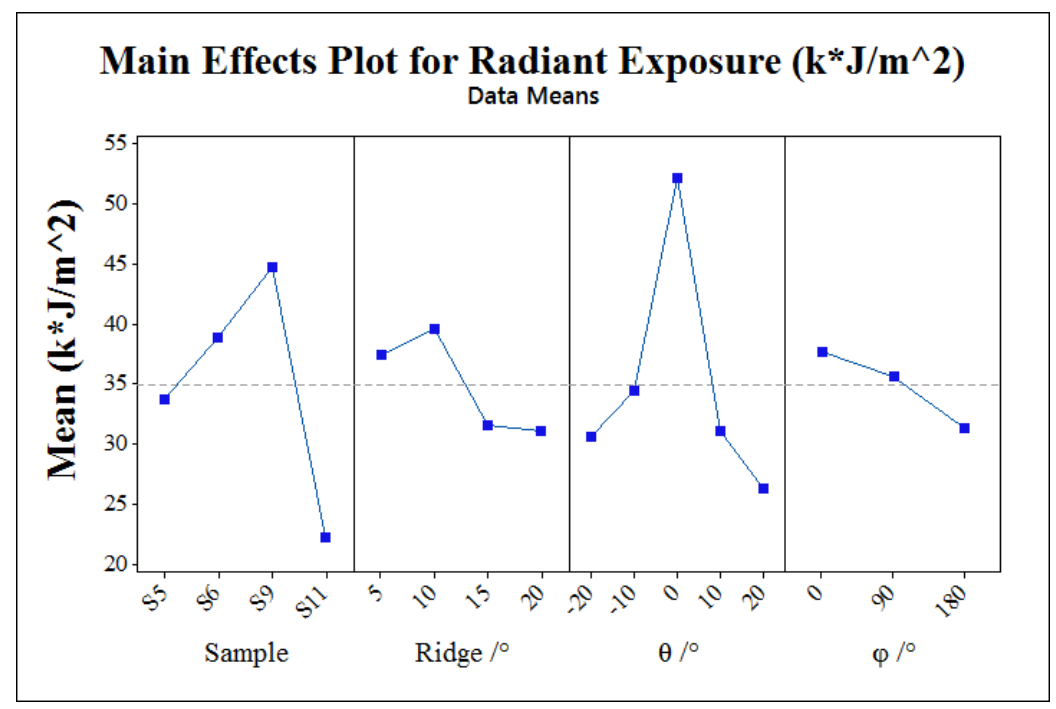

Figure 7. Main effects plot for the absolute reflectance. The name of the samples are listed in table 1. $\theta$ refers to the tilting angle of the microscope. $\varphi$ refers to the azimuthal rotation angle of the sample. 


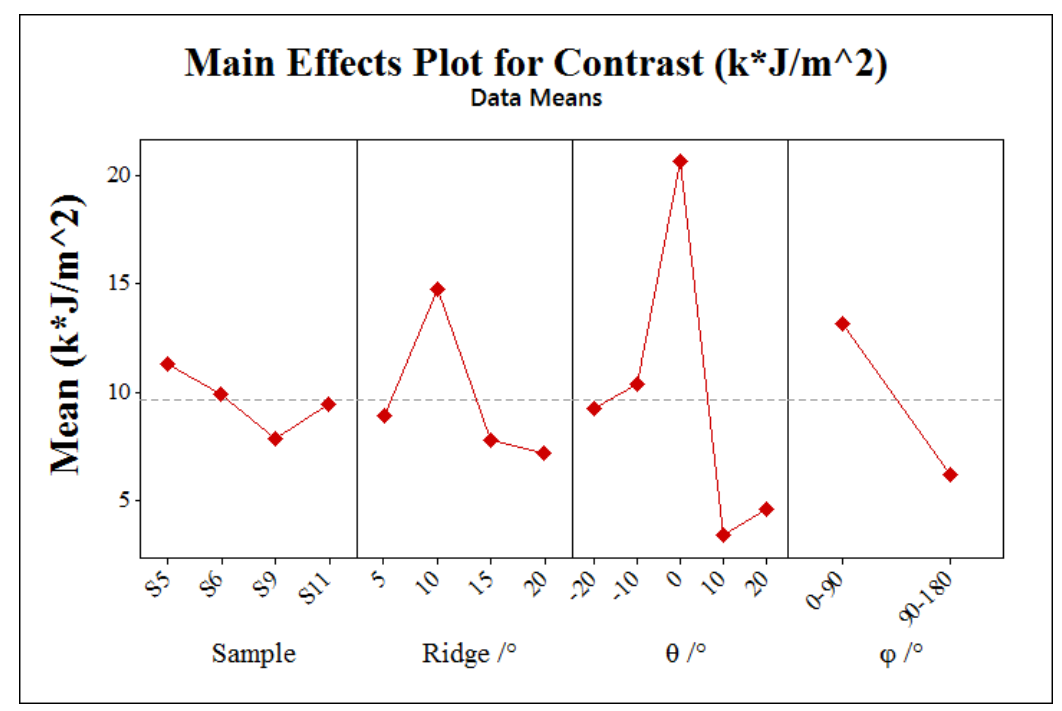

Figure 8. Main effects plot for the contrast. The name of the samples are listed in table 1. $\theta$ refers to the tilting angle of the microscope. $\varphi$ refers to the azimuthal rotation angle of the sample.

\section{Conclusion}

The present study investigated the functionality of a microstructure composed of a close array of ridges, of distance $50 \mu \mathrm{m}$, milled on a steel bar and replicated through hot embossing and silicone replica technology. The purpose was to maximize the reflectance from a certain point of view and minimize it from its orthogonal position. A method involving a Hirox RH-2000 digital microscope was developed. The microscope was used as a gonioreflectometer using calibrated High Dynamic Range (HDR) imaging to extract the radiant exposure of each surface at varying light and camera positions.

The method was able to successfully evaluate both metal and plastic samples. It was noticed that the uncertainty related to the measurements varied from as low as $1 \%$ of the average reflectance for low reflective surfaces, to $20 \%$ for a highly reflective surfaces. Regarding the plastic sample, the 10 degrees ridge and $\mathrm{ABS}$ produced the best results in term of contrast. The latter was also enhanced by darker colours of the materials.

\section{Acknowledgments}

This paper reports work undertaken in the context of project 5163-00001B funded by Innovation Fund Denmark, in a joint research between the Department of Mechanical Engineering and the Department of Applied Mathematics and Computer Science at the Technical University of Denmark.

\section{References}

[1] Bruzzone A A G, Costa H L, Lonardo P M and Lucca D A 2008 Advances in engineered surfaces for functional performance CIRP Ann. - Manuf. Technol. 57 750-69

[2] Chen X, Li H L, Cao H Y, Wharton J, Allanson D and Hu Z Q 2016 Grinding for Microstructural Functional Surface Adv. Mater. Res. 1136 48-53

[3] Flucke C, Gläbe R and Brinksmeier E Diamond Micro Chiseling of Molding Inserts for Optical Micro Structures

[4] Bekesi J, Kaakkunen J J J, Michaeli W, Klaiber F, Schoengart M, Ihlemann J and Simon P 2010 Fast fabrication of super-hydrophobic surfaces on polypropylene by replication of short-pulse laser structured molds Appl. Phys. A Mater. Sci. Process. 99 691-5

[5] Schift H, Urwyler P, Kristiansen P M and Gobrecht J 2014 Nanoimprint lithography process chains for the fabrication of micro- and nanodevices J. Micro/Nanolithography, MEMS, MOEMS 1331303

[6] Hansen H N, Hocken R J and Tosello G 2011 Replication of micro and nano surface geometries CIRP Ann. - Manuf. Technol. 60 695-714 
[7] Theilade U A and Hansen H N 2007 Surface microstructure replication in injection molding Int. J. Adv. Manuf. Technol. 33 157-66

[8] Bruzzone A A G and Costa H L 2013 Functional characterization of structured surfaces for tribological applications Procedia CIRP 12 456-61

[9] Graham E, Park C I and Park S S 2013 Fabrication of micro-dimpled surfaces through micro ball end milling Int. J. Precis. Eng. Manuf. 14 1637-46

[10] Attia U M, Marson S and Alcock J R 2009 Micro-injection moulding of polymer microfluidic devices Microfluid. Nanofluidics 7 1-28

[11] Urwyler P, Schift H, Gobrecht J, Häfeli O, Altana M, Battiston F and Müller B 2011 Surface patterned polymer micro-cantilever arrays for sensing Sensors Actuators, A Phys. 172 2-8

[12] Saito A 2011 Material design and structural color inspired by biomimetic approach Sci. Technol. Adv. Mater. 1264709

[13] Schulz U 2006 Review of modern techniques to generate antireflective properties on thermoplastic polymers. Appl. Opt. 45 1608-18

[14] Kristensen A, Clausen J, Christiansen A B, Højlund-nielsen E, Nielsen C H, Fogh C, Kristiansen T, Simonsen E L, Høvsgaard P and Mortensen N A 2013 Nanostructured optical polymer surfaces by imprinting and injection moulding $14-5$

[15] Weyrich T, Peers P, Matusik W and Rusinkiewicz S 2009 Fabricating microgeometry for custom surface reflectance ACM Trans. Graph. 281

[16] Tawfick S, De Volder M, Copic D, Park S J, Oliver C R, Polsen E S, Roberts M J and Hart A J 2012 Engineering of micro- and nanostructured surfaces with anisotropic geometries and properties $A d v$. Mater. 24 1628-74

[17] Li D, Zhang Y, Regi F, Tosello G, Madsen M H, Nielsen J B, Aanæs H and Frisvad J R 2017 Process chain for fabrication of anisotropic optical functional surfaces on polymer components euspen's 17 th Int. Conf. 4-5

[18] Levin A, Glasner D, Xiong Y, Durand F, Freeman W, Matusik W and Zickler T 2013 Fabricating BRDFs at high spatial resolution using wave optics ACM Trans. Graph. 32 144:1-14

[19] Goodall R H, Darras L P and Purnell M A 2015 Accuracy and Precision of Silicon Based Impression Media for Quantitative Areal Texture Analysis Sci. Rep. 510800

[20] Rusinkiewicz S.M. 1998 A New Change of Variables for Efficient BRDF Representation. Drettakis, G., and Max, N., editors, Rendering Techniques '98. Eurographics. Springer.

[21] Debevec P E and Malik J 1997 Recovering high dynamic range radiance maps from photographs Proc. 24th Annu. Conf. Comput. Graph. Interact. Tech. - SIGGRAPH '97 369-78

[22] ISO 2008 JCGM 100:2008 Int. Organ. Stand. Geneva ISBN 50134 